\title{
Fiber Optic Sensing System for Monitoring of Current Collectors and Catenary of Railways
}

\author{
Kerstin Schröder ${ }^{1}$, Manfred Rothhardt ${ }^{1}$, Wolfgang Ecke ${ }^{1}$, Uwe Richter ${ }^{2}$, André Sonntag ${ }^{2}$, \\ Hartmut Bartelt ${ }^{1}$ \\ ${ }^{1}$ Leibniz Institute of Photonic Technology, Albert-Einstein-Str. 9, 07745 Jena, Germany \\ ${ }^{2}$ Eurailscout Inspection \& Analysis b.v., Torellstr. 1, 10243 Berlin. Germany
}

\section{Summary}

Fiber optic sensors are excellent tools to use for monitoring purposes on high voltage current collectors. Because of their small cross section and electrical neutrality, they are easy to integrate into the current collector strip and are well specialized for detection of high-speed load events. Also the conventional contact force measurement with four force sensors below the collector strips can be simplified by using fiber optic force and acceleration sensors.

\section{Keywords: Force Sensors, Structural Health Monitoring, Current Collectors, Fiber Bragg Gratings}

\section{Introduction}

This work aims at new needs for diagnostics on trains and electrical infrastructure arising from the deregulation and interoperability of the EU railway track. The contact between overhead contact lines (OCLs) and current collectors/pantographs is an interface between the newly established track and train operators. Permanent monitoring of the load conditions allows prediction of the resulting wear and helps to optimize service and repair cycles on the OCL.

Some measurement techniques for $\mathrm{OCL}$ monitoring, such as contact force measurement, are already well established. Boundary conditions are defined in an European standard (EN 50317). Other techniques, for instance the detection of disturbances (misalignments or intruders), are still under development or under optimization. Measurements at the interface between OCLs and current collectors with electrical sensors are complex and prone to interferences due to the high electrical voltages and the strong changes of electric and magnetic fields. In contrast, fiber optic sensors, which have advantages as small and electrically immune devices, can easily be applied for such measurements. For this reason research is currently underway to utilize fiber optic sensors for monitoring purposes in current collectors or on OCLs, already in early stage ([1], [2], [3]).

Fiber Bragg grating (FBG) sensors have the additional advantage that many sensor elements can be multiplexed along a single optical fiber, and the quantitative load result is derived from a spectral shift, which is immune to light intensity variations. Therefore, they are often the preferred kind of sensors for monitoring purposes on this interface ([4], [5]). Currently, such investigations are focused on the optimization of the sensors and their instrumentation. In this paper, results are reported from the investigation of two different measuring techniques: quantitative logging of fast hit events, and of the stationary acceleration-compensated contact force (Fig. 1).

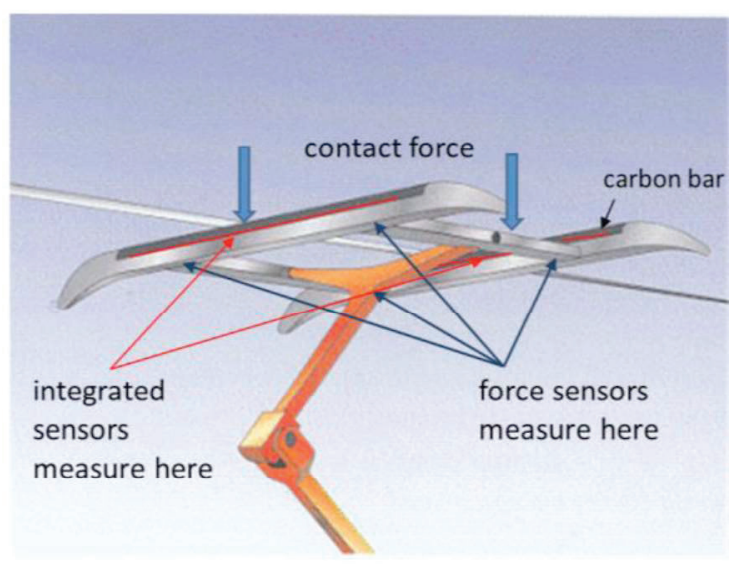

Fig. 1: Pantograph head with overhead contact line, sensitized collector strip carbon und aluminum part of the strip and force sensors below the collector strip. 


\section{FBG Sensor Interrogating System}

There are different ways to interrogate load results from FBG sensors. Our sensor system is based on a broadband light source, and a line-based compact spectrometer with photodetector line, comprising a depolarization component, fiber-optic connectors to four sensor lines, fast and low noise DSP electronics, Ethernet data communication, and a break-free power supply. All these components are placed in a box with dimensions of $18 \mathrm{~cm} \times 28 \mathrm{~cm} \times 10 \mathrm{~cm}$, allowing easy integration into a locomotive (Fig. 2). It can simultaneously read 32 FBG sensors at 5000 measurements per second, with a strain resolution of about $1 \mu \mathrm{m} / \mathrm{m}$ per sensor.

In order to maintain the position of measured events along the rail track, the position data of the locomotives are read directly into the interrogator's DSP, and saved simultaneously with the load data.

For stabilization of long-term contact force measurements, two fiber optic depolarizers (Lyot type at the light source, and a spatial mode mixer in front of the spectrometer, respectively) minimize the influences of FBG birefringence and polarization dependencies of the spectrometer. Each sensor head comprises a FBG force sensor and a FBG acceleration sensor, which are integrated along a single optical fiber.

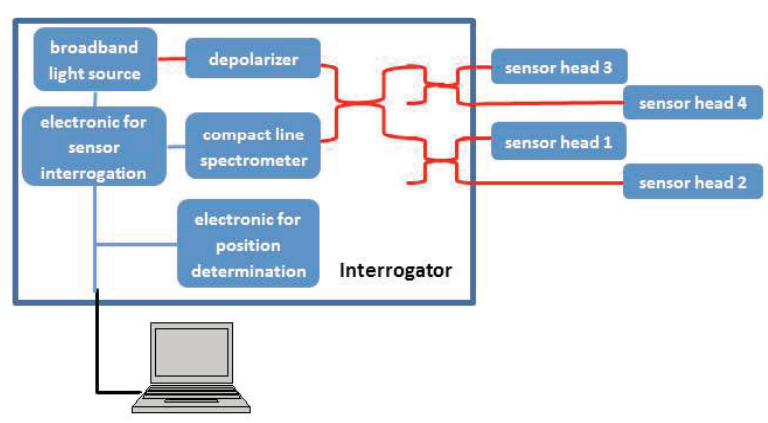

Fig. 2: $\quad$ FBG sensor interrogating system.

\section{Integrated Collector Strip Sensors}

Because of their convenient dimensions, it is comparatively simple to integrate fiber optic sensors, and especially fiber Bragg gratings, inside of the current collector itself. In this case the sensors are very close to the interacting interface collector strip - contact line. The closer the sensors to the interface, the more accurate and free of delays and damping the monitoring of fast hits can be performed.
A first concept tried to use the collector strip as a bending beam, in order to transform also stationary contact forces into strain, which can be measured. The main disadvantage resulted from the low thermal conductivity of the upper strip material (carbon). As a consequence local heating caused from sunshine or high electrical current leads to deformations of the strip, which is detected much earlier by the strain sensors, compared to the true temperature change. The resulting errors are discussed in detail in [1]. As a consequence, the contact force measurement is done with additional fiber optic force sensors fixed below the collector strips. They are described in detail in the chapter 'Contact Force Measurement'.

The collector strip with integrated FBG sensors (shown schematically in Fig. 3) has provided outstanding application results for the detection and measurement of hard hits on the carbon bar in the driving direction. Maladjusted catenary elements or intruders into the catenary region cause these hits. They can damage the carbon of the collector strip and cause serious traffic incidents.

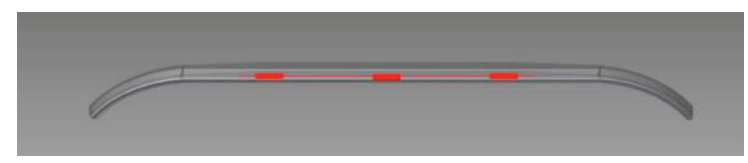

Fig. 3: Collector strip with schematic representation of integrated fiber optical sensors (red). They are positioned in the boundary between aluminum profile and carbon bar, close to the edge, and were used successfully to detect hits in a horizontal (driving) direction.

The detection of low level hits (already below damage threshold) is the aim of this sensing system, in order to provide timely maintenance on demand.

'Soft hits' can be caused by short-term locomotive accelerations, e.g., from rail steps, or vibration of the pantograph on the catenary. Vibrations of the pantograph-catenary system often follow them. Typical frequencies are 220 to $250 \mathrm{~Hz}$ with corresponding peak rise times of $\sim 1 \mathrm{~ms}$ and low damping. These 'soft hits' can also be measured with acceleration sensors below the collector strips.

In contrast, 'hard hits' are characterized by much shorter rise times (50 to $200 \mu \mathrm{s})$ and strong attenuation (an example is given in Fig. 4). They can cause a strong reaction on the embedded strain sensors, which is only detectable with a correspondingly fast measurement system. 
The example in Fig. 4 is measured under laboratory conditions: a hit on the carbon bar is excited by a metal test pendulum. The hard hit on the carbon excites transversal and longitudinal surface waves, which spread at velocities of $3.3 \times 10^{3} \mathrm{~m} / \mathrm{s}$ and $5.7 \times 10^{3} \mathrm{~m} / \mathrm{s}$, respectively, along the carbon bar, and are reflected at its free ends.

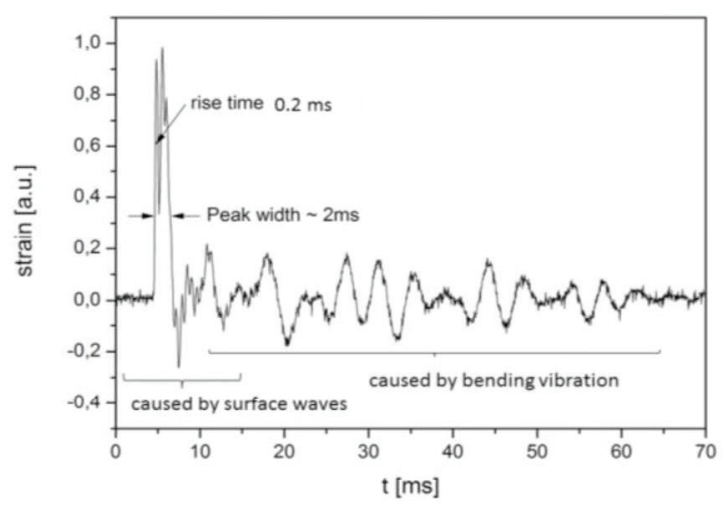

Fig. 4: Sensor response from a hit with a metal pendulum on the carbon bar.

These 'Hard hits' can usually not be detected by sensors far from the interface, below the collector strips, but with integrated sensors only. The sensor system must interrogate at data rates of at least 5000 measurements/s. More detailed laboratory investigations of hit responses have been performed with 20,000 measurements/s.

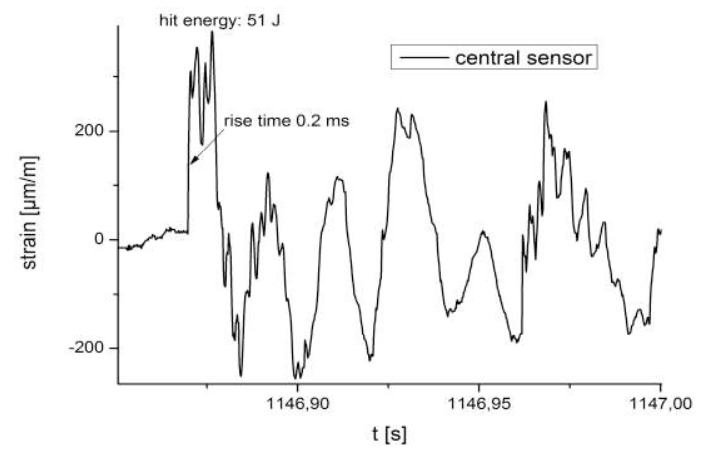

Fig. 5: Signal detected during a test run in Schwarze Pumpe. Passing a section insulator at $37 \mathrm{~km} / \mathrm{h}$ caused this hit. The short rise time is typical for a hard hit. The energy of the hit was estimated to be $51 \mathrm{~J}$.

From such laboratory tests, the dependencies of signal amplitude and hit energy have been found to be linear for these collector strips, with a slope of $7.4 \mu \mathrm{m} / \mathrm{m} / \mathrm{J}$ [4]. This sensitivity depends slightly on the hit position along the collector strip. Since position detection was not yet integrated in this specific system, a slight systematic error occurs for the energy estimation, as in the example from a test run in Schwarze Pumpe in Fig. 5.

\section{Contact Force Measurement}

A usual way to measure a contact force between a current collector and an OCL is the application of force sensors into the fixations of the collector strips. For correction of inertial forces, each force sensor is closely accompanied by an acceleration sensor in the vertical direction. In this work we have implemented such sensors and additionally developed force and acceleration sensors using fiber optic Bragg grating sensors.

\section{Contact Force Sensors}

The contact force sensors are made as S-type force sensors, as schematically shown in Fig. 7a. To achieve a high sensitivity it is necessary to choose a material with very high elasticity limit Rp0.2. We tested spring brass as well as steel type NivaFlex $45 / 18$ with Rp0.2 of 500 and $1800 \mathrm{MPa}$, respectively.
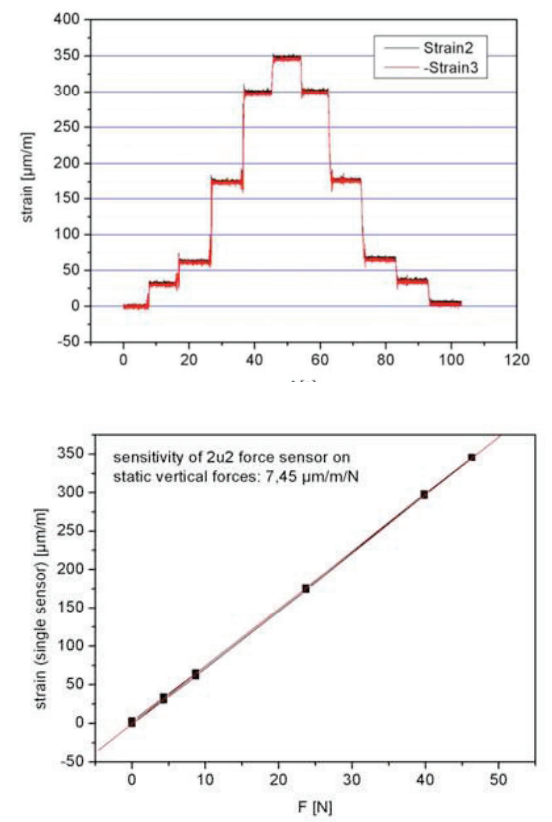

b)

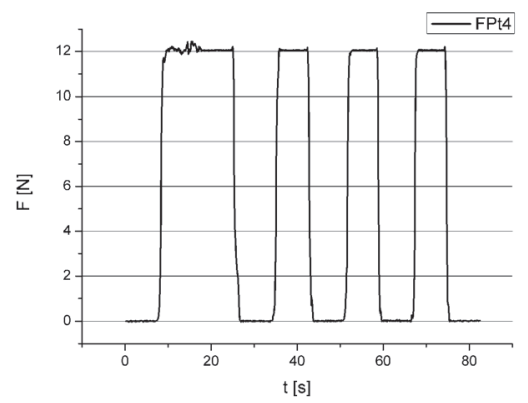

c)

Fig. 6: a) Force dependence of a single force sensor and

b) The resulting sensitivity.

c) Reproducibility of the force measurement. The sensor is loaded and deloaded four times with a $12 \mathrm{~N}$ mass. 
The sensitivity was tested via loading and deloading the sensor with defined masses. An example measurement for the spring brass sensor is shown in Fig. 6a and 6b.

A sensitivity of $7.45 \mu \mathrm{m} / \mathrm{m} / \mathrm{N}$ was achieved. With the steel NivaFlex $45 / 18$ a sensitivity of $22 \mu \mathrm{m} / \mathrm{m} / \mathrm{N}$ was achieved.

This allows a measurement range up to $200 \mathrm{~N}$ with a safety factor of 1.8 for the elastic deformation region. Fig. $6 c$ shows the result of a reproducibility experiment with a single NivaFlex based force sensor.

A test mass of $12 \mathrm{~N}$ was positioned on the sensor (the reaction on the positioning movements are visible on the changeovers) and removed four times. The reproducibility error of both the $12 \mathrm{~N}$ and the $0 \mathrm{~N}$ measuring values are below $0.1 \mathrm{~N}$.

In general, the readout of strain sensors is cross sensitive to temperature and thus a specific concept is necessary for its compensation. The use of an anti-symmetric sensor, with equal strain but opposite sign (Fig. $7 a$ ), is used in this concept. Force is calculated from the difference in the readouts of both sensors while temperature is calculated from their sum.

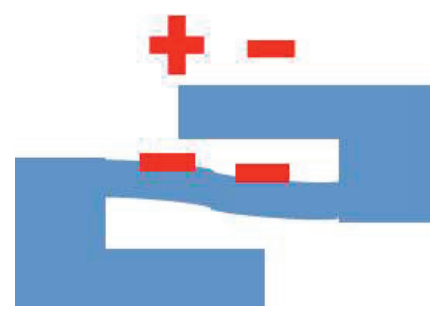

a)

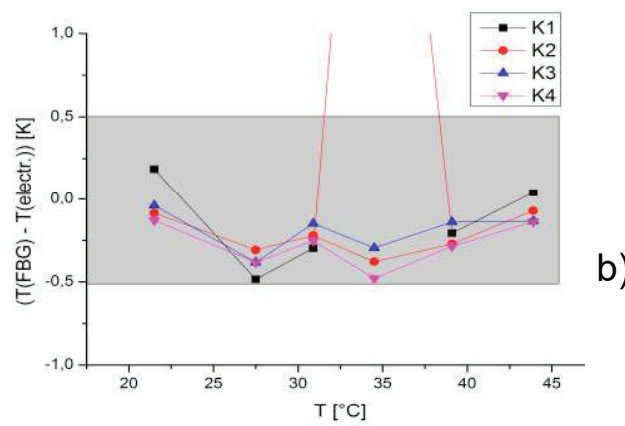

Fig. 7: a) Concept for temperature compensation.

b) Accuracy of temperature measurement with four sensor heads in an oven at temperatures between 20 to $45{ }^{\circ} \mathrm{C}$, in comparison to electrical temperature sensors.

\section{Acceleration Sensors}

In order to compensate the measured force values for inertial forces, acceleration sensors are added to the contact force sensor heads.
Different types of FBG based acceleration sensors can be found in the literature. [6] and [7] stretch a FBG by a moving mass. [8] used a bending beam. [9] investigated a cantilever based FBG accelerometer.

In our work we developed two different types of cantilever based FBG accelerometers. Both of them used bending beams, which are bent by the reaction of an inertial mass to acceleration. The bending beam was triangular in design in order to generate a constant strain distribution. Prototype \#as1 used a spring steel - bending beam with a FBG mounted $2 \mathrm{~mm}$ above the surface and a mass of $60 \mathrm{~g}$ fixed on the tip of the triangular bending beam.

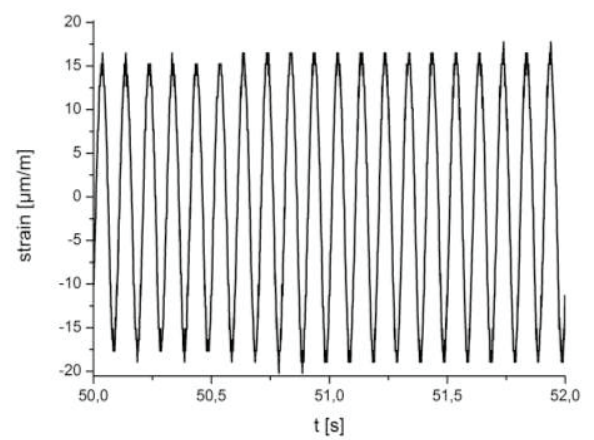

a)

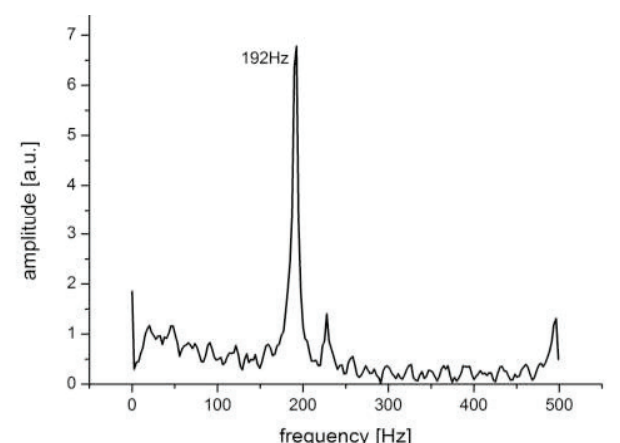

b)

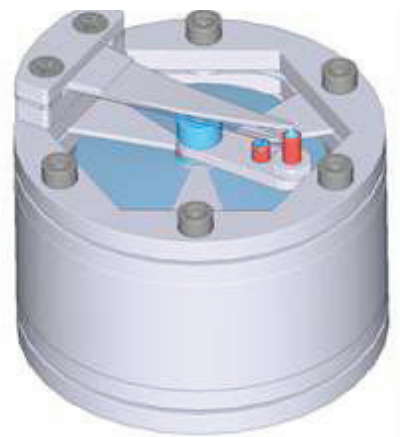

c)

Fig. 8: a) Reaction of sensor \#as1 to a sinusoidal excitation of frequ. $10 \mathrm{~Hz}$. b) Resonance frequency of sensor \#as1 is $192 \mathrm{~Hz}$.

c) Design of sensor \#as2. The mass of about $50 \mathrm{~g}$ was fixed with steel springs and it moved the triangular bending beam on top, where the FBG sensor is attached. 
For the second prototype (\#as2), the mass was fixed with steep springs, and it moves an additional steel triangle with an attached FBG (see Fig. 8c). In this configuration, the mass holder and the sensor-bending beam can be optimized separately.

Both types were tested for their sensitivities and resonance frequencies with help of a Shaker (TIRA S 51128). For \#as1, a sensitivity of $36 \mu \mathrm{m} / \mathrm{m} / \mathrm{G}$ and a resonance frequency of $192 \mathrm{~Hz}$ was measured. For \#as2, the sensitivity was strongly dependent on the fine adjustment. Sensitivities were measured in the range of $24 \mu \mathrm{m} / \mathrm{m} / \mathrm{G}$ up to $72 \mu \mathrm{m} / \mathrm{m} / \mathrm{G}$. The lowest resonance is weak, but at a very low frequency of approximately $86 \mathrm{~Hz}$.

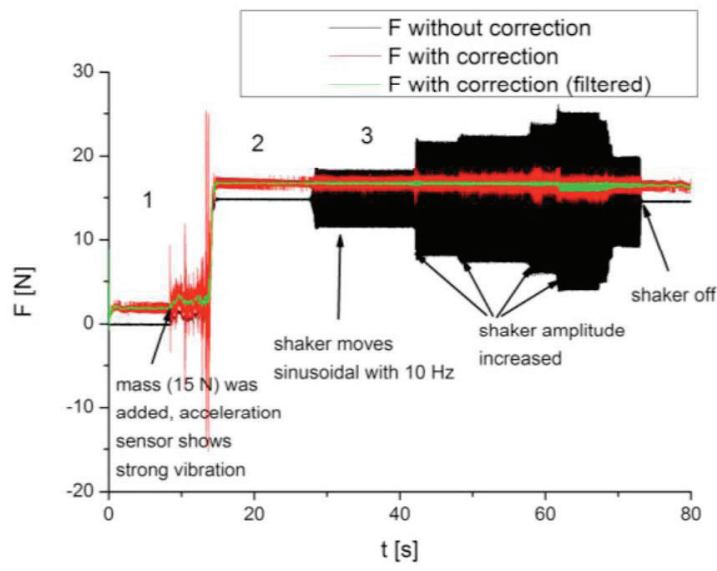

Fig. 9: Contact force sensor with acceleration sensor in test on a shaker.

black: Contact force results without acceleration compensation (very low noise during stand-still)

red: Contact force results with acceleration compensation ( 35 $\mu \mathrm{m} / \mathrm{m} / \mathrm{G}$ and $190 \mathrm{~Hz}$ ). An offset was added in the graphic.

green: Characteristic in red, plus $20 \mathrm{~Hz}$ low pass filter (corresponding to EN50317)

To check the performance of the acceleration sensor, and to test the compensation of the inertial force of the force sensor, both sensors were vibrated by the shaker. The force sensor was loaded with a mass of $15 \mathrm{~N}$. Results of an example measurement are given in Fig. 9. In segment 1 the system is initially undisturbed. The fixation of the $15 \mathrm{~N}$ mass then excites a strong high frequency disturbance on the system. This cannot be compensated by the acceleration sensor (red curve). However, it is eliminated after application of a $20 \mathrm{~Hz}$ lowpass filter, as it is mandatory in European standard EN50317. In segment 2, the mass was applied, and the sensor was not moved. In segment 3 the shaker moved the sensor by sinusoidal waveform at a frequency of $10 \mathrm{~Hz}$. The red curve (contact force with compensation of inertial force) is expected to be smooth in this section, and these expectations are fulfilled very well. After the mandatory filtering, a residual deviation from the $15 \mathrm{~N}$ set value was kept within $+/-0.5 \mathrm{~N}$.

\section{Sensor Integration}

The interface between the force sensor and the collector strip is being developed by a third party. It fulfills the task of negligible low cross sensitivities to further forces on the collector strip. The task of reproducible transition of the contact force from the collector strip to the sensor has not yet been solved by this technique. A revision is under preparation. Therefore the reproducibility of the whole system in dependence of dynamic force frequencies cannot yet be evaluated in the actual status.

\section{First Test Drive}

A first test drive was done to show the robustness and performance of the sensing system. It took place on a track of the Lignitefired power plant in Schwarze Pumpe in Germany.
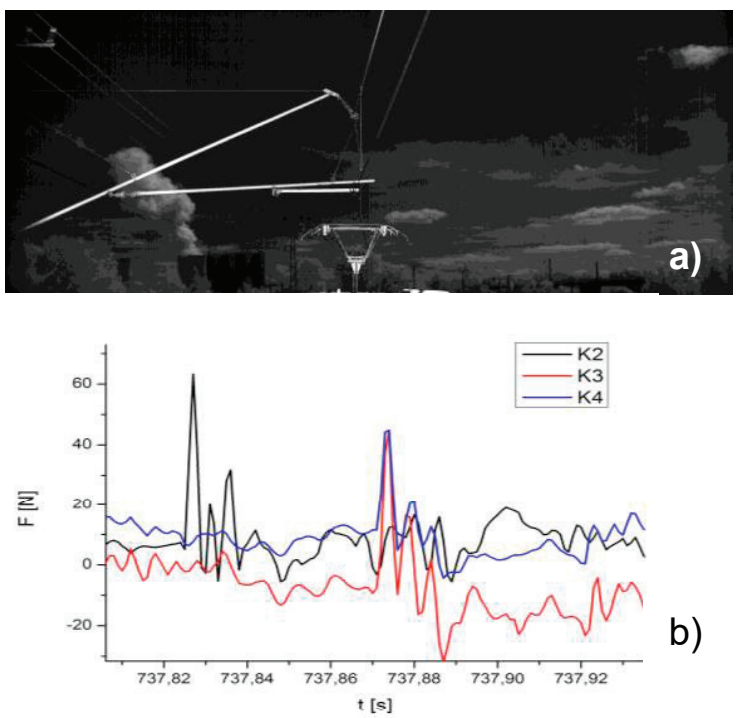

Fig. 10: a) Test drive in 'Schwarze Pumpe'; b) A week 'event' was measured firstly by the collector strip above sensor head K2, and after $40 \mathrm{~ms}$ it is detected by the sensor heads $K 3$ and K4, which are mounted below the trailing, second collector strip of the pantograph head. 
Three sensor heads were mounted below the collector strips (the fourth one was damaged shortly before the tests). Signal transmission was done with fiber optic cables directly from the pantograph on $15 \mathrm{kV}$ high voltages to the interrogator in the driver's cabin. To avoid creepage currents on a dirty and wet cable surface, a $20 \mathrm{~cm}$ long section of the cables was embedded in a 'creepage path elongation'.

To correlate the measured events with track positions, a position signal was given from the locomotive electronics to the interrogator and was saved simultaneously with the measured force values.

A video system monitored the pantograph and the catenary (Fig. 10a) during part of the test drive. From the video, a number of events were easily correlated to special catenary elements.

This study focused on significant force changes only, due to the non-optimized force sensor connection below the collector strips. However, force changes have been measured relative to a specified reference force value, which was set to the sensor system while the pantograph head was pressed to the catenary and the locomotive halted.

In Fig. 10b an example is given, with the locomotive passing a section insulator. The event occurs firstly on sensor K2, which was attached below the leading collector strip. After $50 \mathrm{~ms}$ the same event was measured by sensor heads $\mathrm{K} 3$ and $\mathrm{K} 4$, which are mounted below the trailing collector strip. The strips are $60 \mathrm{~cm}$ apart and the locomotive was travelling at speed of $43 \mathrm{~km} / \mathrm{h}$.

\section{Acknowledgement}

This work is supported by Freistaat Thüringen (project no. TNA I-1/2012), co-financed by the European Funds for Regional Development (EFRE), the AiF ZIM (project no. KF2206912DB3) and Eurailscout Inspection \& Analysis b.v. The authors thank their supporters very much.

\section{References}

[1] K. Schröder, W. Ecke, M. Kautz, S. Willett, M. Jenzer, An approach to continuous on-site monitoring of contact forces in current collectors by a fiber optic sensing system, Optics and Lasers in Engineering 51 (2), 172-179 (2012); doi:10.1016/j.optlaseng.2012.08.007

[2] N. Theune, T. Bosselmann, M. Willsch, R. Puschmann, Optical monitoring of highly stressed infrastructure and rolling stock modules for railway system operation, Proceedings of SPIE 5502 55022S-1-4 (2004); DOI: $10.1117 / 12.566693$
[3] P. Boffi, G. Cattaneo,L. Amoriello, A. Barberis, G. Bucca, M. Bocciolone, A. Collina Optical fiber sensors to measure collector performance in the pantograph-catenary interaction, IEEE Sensors Journal 9, 635-40 (2009); doi:10.1109/JSEN.2009.2020244

[4] K. Schröder, W. Ecke, M. Kautz, S. Willett, H. Unterwaditzer, T. Bosselmann, M. Rothhardt, Smart current collector-fibre optic hit detection system for improved security on railway tracks, Measurement Science and Technology 24 (11), 1-9 (2013); doi:10.1088/09570233/24/11/115104

[5] L. Comolli, G. Bucca, M. Bocciolone, A. Collina, First results from in line strain measurements with FBG sensors on the pantograph collector of underground trains, Proc. Of SPIE 7726, 772605-1-4 (2010); doi:10.1117/12.854395

[6] P. Antunes, H. Varum, P. André, Uniaxial fiber Bragg grating accelerometer system with temperatureand cross axis insensitivity, Measurement 44, 55-59 (2011), doi:10.1016/j.measurement.2010.09.013

[7] Q. Jiang, M. Yang, Simulation and experimental study of a three-axis fiber Bragg grating accelerometer based on the pull-push mechanism, Meas. Sci. Technol. 24, 1-9, (2013); doi:10.1088/0957-0233/24/11/115105

[8] A. Mita, I. Yokoi, Fiber Bragg grating accelerometer for buildings and civil infrastructures, Proceedings of SPIE 4330, 479486 (2001); doi:10.1117/12.434148

[9] H. Y. Au, S. K. Khijwania, H. Y. Tam, Fiber Bragg Grating Based Accelerometer, Proc. of SPIE 7004 70042S-1-4 (2008); doi: $10.1117 / 12.785992$ 